\title{
Gut microbiota in experimental murine model of Graves' orbitopathy established in different environments may modulate clinical presentation of disease
}

Giulia Masetti ${ }^{1,2}$, Sajad Moshkelgosha ${ }^{3,4,5}$, Hedda-Luise Köhling ${ }^{6,7}$, Danila Covelli,8, Jasvinder Paul Banga 3,4, Utta Berchner-Pfannschmidt ${ }^{3}$, Mareike Horstmann³ ${ }^{3}$ Salvador Diaz-Cano ${ }^{9}$, Gina-Eva Goertz ${ }^{3}$, Sue Plummer ${ }^{6}$, Anja Eckstein ${ }^{3}$, Marian Ludgate ${ }^{1}$, Filippo Biscarini ${ }^{1,2,10}$, Julian Roberto Marchesi ${ }^{11,12^{*}}$ and the INDIGO consortium ${ }^{13}$

\begin{abstract}
Background: Variation in induced models of autoimmunity has been attributed to the housing environment and its effect on the gut microbiota. In Graves' disease (GD), autoantibodies to the thyrotropin receptor (TSHR) cause autoimmune hyperthyroidism. Many GD patients develop Graves' orbitopathy or ophthalmopathy (GO) characterized by orbital tissue remodeling including adipogenesis. Murine models of GD/GO would help delineate pathogenetic mechanisms, and although several have been reported, most lack reproducibility. A model comprising immunization of female BALBC mice with a TSHR expression plasmid using in vivo electroporation was reproduced in two independent laboratories. Similar orbital disease was induced in both centers, but differences were apparent (e.g., hyperthyroidism in Center 1 but not Center 2). We hypothesized a role for the gut microbiota influencing the outcome and reproducibility of induced GO.

Results: We combined metataxonomics (16S rRNA gene sequencing) and traditional microbial culture of the intestinal contents from the GO murine model, to analyze the gut microbiota in the two centers. We observed significant differences in alpha and beta diversity and in the taxonomic profiles, e.g., operational taxonomic units (OTUs) from the genus Lactobacillus were more abundant in Center 2, and Bacteroides and Bifidobacterium counts were more abundant in Center 1 where we also observed a negative correlation between the OTUs of the genus Intestinimonas and TSHR autoantibodies. Traditional microbiology largely confirmed the metataxonomics data and indicated significantly higher yeast counts in Center 1 TSHR-immunized mice. We also compared the gut microbiota between immunization groups within Center 2, comprising the TSHR- or ßgal control-immunized mice and naïve untreated mice. We observed a shift of the TSHR-immunized mice bacterial communities described by the beta diversity weighted Unifrac. Furthermore, we observed a significant positive correlation between the presence of Firmicutes and orbital-adipogenesis specifically in TSHR-immunized mice.

(Continued on next page)
\end{abstract}

\footnotetext{
* Correspondence: MarchesiJR@cardiff.ac.uk

Filippo Biscarini and Julian Marchesi these authors are joint senior author.

Giulia Masetti and Sajad Moshkelgosha these authors contributed equally to the study.

${ }^{11}$ School of Biosciences, Cardiff University, Sir Martin Evans Building, Museum

Avenue, Cardiff CF10 3AX, UK

${ }^{12}$ Center for Digestive and Gut Health, Imperial College London, W2 1NY,

London, UK

Full list of author information is available at the end of the article
}

(c) The Author(s). 2018 Open Access This article is distributed under the terms of the Creative Commons Attribution 4.0 International License (http://creativecommons.org/licenses/by/4.0/), which permits unrestricted use, distribution, and reproduction in any medium, provided you give appropriate credit to the original author(s) and the source, provide a link to the Creative Commons license, and indicate if changes were made. The Creative Commons Public Domain Dedication waiver (http://creativecommons.org/publicdomain/zero/1.0/) applies to the data made available in this article, unless otherwise stated. 


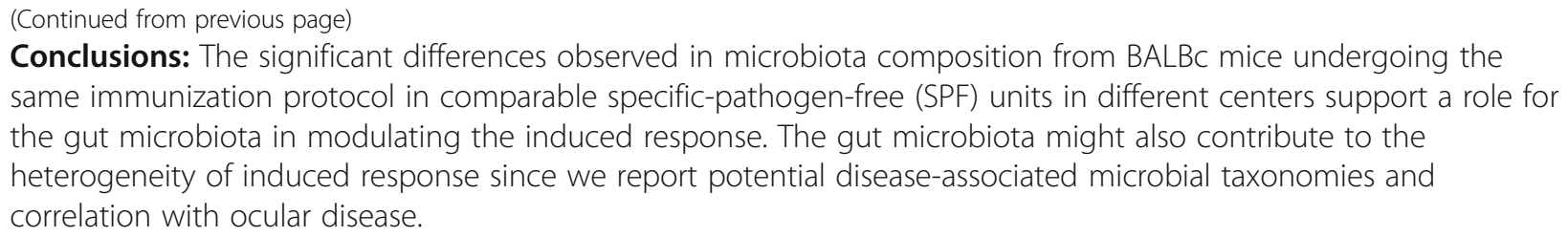

Keywords: Graves' orbitopathy, Graves' disease, Induced animal model, Gut microbiota, TSHR, Metataxonomics, Orbital adipogenesis, Firmicutes

\section{Background}

The poor reproducibility of murine models of human diseases has become a puzzling phenomenon in recent decades. Apart from the genetic background of the strains used, the type of animal housing, diet, and even the vendor can influence disease phenotype [1, 2].

In Graves' disease (GD) and Graves' orbitopathy or ophthalmopathy (GO), in vivo models could help to unravel the pathogenetic mechanisms leading to autoimmunity and identify new therapeutic targets [3]. The lack of spontaneous models of GD and GO necessitates induction of disease under laboratory conditions (reviewed in [4]).

Graves' disease is an organ-specific antibody-mediated autoimmune disease, governed by both genetic predisposition and environmental factors, in which thyroid-stimulating antibodies (TSAb) mimic the function of thyroid-stimulating hormone (TSH) to activate the thyrotropin receptor (TSHR). Moreover, the presence of thyroid-stimulating blocking antibodies (TSBAb), which inhibit the TSHR signaling cascade, and neutral antibodies to TSHR have been described in GD [5]. GD symptoms include hyperthyroidism, weight loss, heat intolerance, and tremors; it affects about $2 \%$ of the population in the UK, with a female predominance. About $20 \%$ of GD patients develop an eye disease, $\mathrm{GO}$, characterized by pro-inflammatory cells and tissue remodeling (extraocular muscle inflammation, adipogenesis, overproduction of extra-cellular matrix) in the orbit [6].

Several GD mouse models have been developed using different immunization protocols with no signs of concomitant eye disease as previously reviewed $[4,7,8]$. Ludgate and colleagues established a TSHR-induced GO model by genetic immunization, i.e., injecting a TSHR expression plasmid [9]. Mice developed thyroiditis plus some aspects of GO and disease could be transferred to naive recipients using the TSHR-primed $\mathrm{T}$ cells from the genetically immunized mice. However, the model could not be reproduced in a different animal unit (neither was specific-pathogen-free (SPF)), and the TSHR-induced disease was quite distinct from that previously described, which the authors postulated might be due to microorganisms [10]. It has also been reported that TSHR-immunized mice from a conventional environment had higher and more persistent TSAb levels than mice in SPF units [11].

Recently, Berchner-Pfannschmidt and colleagues reported the induction of GO-like disease in two independent SPF units [12]. The immunization protocol utilized genetic delivery of TSHR A-subunit plasmid by close field electroporation, which leads to features of GD accompanied by symptoms of eye disease, such as adipogenesis and inflammatory infiltrates in the orbit $[7,13]$. Controls received a plasmid encoding the $\beta$-galactosidase ( $\beta$ gal) gene delivered by the same procedure. Most aspects of the model were reproduced successfully; however, there was heterogeneity in induced disease and differences in thyroid function in the animals undergoing experimental $\mathrm{GO}$ in the two locations [12].

Over the years, the gut microbiota has been associated with several diseases [14-17] and its confounding role in establishing or reproducing disease phenotype in murine models has also been proposed [18].

The murine model of multiple sclerosis, experimental autoimmune encephalomyelitis (EAE), seems to be highly influenced by the gut microbiota. Oral antibiotic immunization and consequent depletion of the gut bacteria, before disease induction, resulted in protection from disease development, along with reduction in pro-inflammatory mediators such as IL-17 and an increased Th2-immune response [19]. On the contrary, the intestinal monocolonization of germ-free mice (sterile) with segmented filamentous bacteria (SFB) restored the disease phenotype, along with an increased number of Th17 cells in the CNS, suggesting a direct interplay of the gut microbiota and the immune response in EAE development [20].

Based on these observations, we hypothesized that the gut microbiota itself might play a major role not only in the establishment but also in the reproducibility of the GO animal model described above. The presence or absence not only of pathogens but also of symbiotic and commensal bacteria can favor an immune response more prone to inflammation and conducive to autoimmunity [21]. 
We aimed to characterize, for the first time, the gut microbiota of the GD/GO models via a combination of metataxonomics (16S rRNA gene sequencing) and traditional microbial culture approaches. We compared the gut contents of TSHR-immunized mice from the two centers to understand whether variation in gut composition could explain differences in the disease induced. Within one center, we then characterized the gut microbiota between different immunizations (TSHR and $\beta$ gal) and compared them with untreated mice, to determine whether the gut microbiota can influence the outcome and correlate with disease features.

\section{Methods}

\section{GO preclinical mouse model samples}

Mouse samples used in the present work were obtained from a recent study [12], conducted in parallel in two independent animal housing units, under comparable SPF conditions. Animal procedures in center 1 were reviewed and approved by the Ethical Committee of King's College London and conducted with Personal and Project licenses under United Kingdom Home Office regulations. Animal procedures in center 2 were reviewed and approved by North Rhine Westphalian State Agency for Nature, Environment and Consumer Protection (LANUV), Germany. Samples from the animal unit of King's College London (UK) will be referred to as "Center 1 " and included a total of 5 TSHR-immunized mice (TSHR). Samples from the University of Duisburg-Essen (Germany) will be referred to as "Center 2", including 10 TSHR-immunized (TSHR), 8 Bgal plasmid controls ( $\beta$ gal), and 6 untreated mice (included as a background control), as shown in Table 1 .

The GO immunization protocol has been previously described [13]. Briefly, 6-8-week-old BALB/cOlaHsd female mice were immunized via intramuscular injection into each biceps femoris muscle [22] and electroporation of either the eukaryotic expression

Table 1 Description of the mouse groups involved in this study

\begin{tabular}{lllll}
\hline No. of animals & Immunization & Centers & Source & Timepoint \\
\hline 5 & TSHR & 1 & Intestinal scraping & T4 \\
10 & TSHR & 2 & I.S./Feces & T0-T4* \\
8 & Bgal & 2 & I.S./Feces & T0-T4* \\
6 & Untreated & 2 & I.S./Feces & T4 $^{\circ}$
\end{tabular}

A total of 23 female BALB/cOlaHsd, 6-8-week-old mice were challenged either with the pTriEx1.1Neo-hTSHR to induce disease (TSHR group) or with pTriEx1.1 Neo- $\beta$-gal as a plasmid control group ( $\beta$ gal group). Independent SPF animal units were based in London (Center 1 ) and Essen (Center 2). An untreated group of six mice has been included as a background control. Samples collection comprised of intestine scraping (I.S.) from Center 1 and both fecal pellets and I.S. within Center 2

*Fecal pellets of $\beta \mathrm{gal}$ and TSHR-immunized mice have been collected before any immunization (TO) and during the time course of the immunization protocol until the sacrifice (T4), as represented in Additional file 1: Figure S1 Untreated mice were sampled at T4 before (fecal) and after the sacrifice (intestinal scraping) plasmid pTriEx1.1Neo-hTSHR (hTSHR289) (TSHR group) or the control plasmid pTriEx1.1Neo- $\beta$-gal (plasmid-control, $\beta$ gal group). All animals, whether TSHR or $\beta$ gal controls, received a total of four plasmid injections at 3 -week intervals of the experiment $(0,3,6$, and 9 weeks).

Each mouse was anesthetized using isoflurane with a properly calibrated vaporizer throughout the immunization procedure (injection and electroporation). After the immunization, mice were carefully transferred to a recovery cage until fully recovered.

Mice in Center 1 were maintained conventionally in open cages in one room and co-housed at a maximum of three animals per cage. In Center 2, the mice were co-housed according to their immunizations, two to four animals per individually ventilated cage in one room. All mice were provided by different outlets of the same supplier (Harlan Ltd. or Harlan laboratories BV). In both centers, mice received autoclaved water and had been fed ad libitum similar commercial chow from different suppliers (Rat and Mouse no.1 Maintenance from Special Diet Services, LBS Biotech UK for Center 1 and Rat/Mouse Maintenance V1534-300 from Ssniff Spezialadiaten $\mathrm{GmbH}$, Germany, for Center 2). Also the cage bedding was from different suppliers.

All immunized and control mice in both locations were sacrificed 9 weeks after the last immunization (18 weeks) to permit the development of the chronic phase of the disease in the TSHR group (Additional file 1: Figure S1).

After sacrifice, murine intestines were snap-frozen and stored in sterile containers at $-80{ }^{\circ} \mathrm{C}$. For subsequent analysis, whole intestines were thawed and directly afterwards placed on a sterile padding. The organs were dissected into two or three parts and the content of all parts was scratched out from oral to aboral end with a sterile inoculation loop resulting in one sample per mouse, which was collected in a sterile container and frozen at $-80{ }^{\circ} \mathrm{C}$ until needed. Within Center 2 only, fecal pellets of $\beta$ gal- and TSHR-immunized mice were also collected before each injection (week 0, 3, 6, and 9). Total DNA was extracted from fecal pellets as described below.

Methods for (i) the evaluation of clinical symptoms, (ii) the determination of the thyroid hormone thyroxine blood levels (fT4) and TRAB (both stimulating TSAb and blocking TSBAb) antibodies, and (iii) the measurement of the expansion of fat cells (adipogenesis) and muscular atrophy in the orbit have been already described [12]. A full description of the mice involved and samples collected in the present study is represented in Table 1 .

Traditional microbial cultures of mouse gut contents A total of 29 scraped intestinal samples (Table 1) derived from Center 1 and Center 2 were analyzed. One gram of 
feces per mouse was diluted in $9 \mathrm{~mL}$ pre-reduced maximum recovery diluent (CM0733, Oxoid, Basingstoke, United Kingdom) with $20 \% v / v$ glycerol and the solution was mixed by vortexing for $5 \mathrm{~s}$. Afterwards, 10-fold serial dilutions were prepared, and $100 \mu \mathrm{l}$ of each dilution was plated onto different culture media under aerobic or anaerobic conditions (Anaerobic Workstation, AW400SG, Elektrotek, Keighley, West Yorkshire, UK). Specific media, culture conditions, and dilution used to isolate different bacteria are listed in Additional file 2.

Bacteria were identified by Gram staining, colony morphology, the presence of spores, and catalase reaction and partially by the API system (BioMerieux, Marcy-l'Étoile, France). Viable bacterial cell counts were enumerated and all counts were recorded as the numbers of $\log 10$ colony forming units per gram of sample. Counts data were Box-Cox transformed before statistical analysis [23]. Mouse groups were compared through the analysis of variance (one-way ANOVA) and Tukey's multiple comparisons test with adjusted $P$ values.

\section{Extraction of total DNA from gut contents and feces and 16S rRNA gene sequencing}

A total of 29 scraped intestinal samples and 96 fecal pellets were individually placed in 2-mL tubes prefilled with $0.1 \mathrm{~mm}$ silica and zirconia bead mix (Benchmark Scientific, Edison, USA), dissolved in $1 \mathrm{~mL}$ InhibitEX buffer (Qiagen Ltd., West Sussex, UK) and vortexed until homogenized. A bead-beating step (Beadbug microcentrifuge homogenizer, Benchmark Scientific, USA) was applied for $3 \times 60 \mathrm{~s}$ at $5 \mathrm{~m} / \mathrm{s}$ with $5 \mathrm{~min}$ rest in-between. The DNA extraction has been performed with QiAmp Fast DNA Stool Mini kit (Qiagen Ltd., UK), following the manufacturer's instruction. Total genomic DNA was eluted in sterile microcentrifuge tubes and quantified by Qubit Fluorimetric Quantitation (ThermoFisher Scientific Ltd., UK), following the manufacturer's instructions. DNA aliquots were kept at $-20{ }^{\circ} \mathrm{C}$ until used. Sequencing of the variable regions of the $16 \mathrm{~S}$ rRNA gene was performed at Research and Testing Laboratory LLC (Lubbock, Texas, USA). Primers used to amplify the $\mathrm{V} 1-\mathrm{V} 2$ regions of $16 \mathrm{~S}$ rRNA gene were 28F (5'-GAGTTTGATCNTGGCTCAG-3') and 388R (5'-TGCTGCCTCCCGTAGGAGT-3'). Sequencing was performed using an Illumina Miseq (Illumina, San Diego, USA), with $10 \mathrm{~K}$ paired-end sequencing protocol.

Processing and statistical analysis of metataxonomic data Processing of the sequences was performed using Mothur v1.36, to reduce possible PCR effects and to cluster sequences into operational taxonomic units
(OTUs) at the $97 \%$ identity cut-off and provide the taxonomic annotations [24]. Paired-end reads (R1 and R2) were joined for each sample using the Mothur function "make.contigs" and trimmed at the 2.5\%-tile and $97.5 \%$-tile on the distribution lengths of the amplicons. Sequences with any ambiguities (i.e., Ns) were removed by setting parameter $N=0$. Filtered sequences were aligned against the SILVA $16 \mathrm{~S}$ rRNA gene reference database (http://www.arb-silva.de). Removal of chimera sequences was done with the Uchime tool [25]; singleton and non-bacterial sequences (e.g., Archaea, eukaryotic, chloroplast, and mitochondria) have been removed from the analysis. The taxonomic assignment from phylum to genus level of the processed sequences was done using the Ribosomal Database Project (RDP) Naïve Bayesian Classifier, using Trainset 14 with a cut-off of $80 \%$ [26]. FastTree (version 2.1.7) has been used to build a phylogenetic tree, using an approximated maximum likelihood solved by Jukes-Cantor evolutionary model [27]. To reduce the effect of possible different sampling methods and to obtain comparable sequencing libraries, each sample library has been subsampled based on the smallest library size. OTUs with less than 10 counts have been excluded from the dataset and grouped as "OTU_low", and the analysis has been performed collapsing OTUs at the phylum-genus levels. Statistical analysis was performed in R (Version 3.2.2) and STAMP tool for metataxonomic data analysis [28].

Alpha diversity indices (observed OTUs, Chao1, ACE, and Shannon) were calculated within samples from Mothur and tested for association with covariates (e.g., locations and immunizations) using a linear model, followed by Tukey's honest significant difference (HSD) post hoc analysis.

Beta diversity was estimated using the Unifrac weighted distance to compare bacterial communities among samples [29], and represented in a non-metric dimensional scaling (NMDS) plot. The permutational multivariate analysis of variance (PERMANOVA) was calculated through the Adonis function [30] in $R$ Vegan package (using 999 permutations) and was used to test the association between the microbiota composition and the covariates (e.g., location of the laboratories or immunizations).

The hierarchical clustering of genera was performed using the Spearman distance and the Ward agglomeration method. Statistical tests with $P \leq 0.05$ were considered as significant.

Over multiple timepoints, the effects of time, immunizations, and their interactions have been estimated on the fecal microbiota composition, all by means of the following linear model: 


$$
\begin{aligned}
y_{i j k}= & \mu+\text { Time }_{i}+\text { Immunization }_{j} \\
& +\left(\text { Time } \times{\text { Immunization })_{i j}}+e_{i j k}\right.
\end{aligned}
$$

where $y$ is the vector of either alpha diversity Chao or Shannon indices, or of the Firmicutes/Bacteroidetes ratio calculated from the relative abundances in each sample at each timepoint; $\mu$ is the overall mean; time is the effect of timepoint in classes (T0, T1...T4); immunization is the type of immunization (either the TSHR or $\beta$ gal). The factorial interaction between immunization and time has also been included in the model; $e$ is the vector of residual effects. Comparison between $\beta$ gal and TSHR immunizations at each timepoint was made using the pairwise $T$ test with Benjamini-Hochberg correction for false discovery rate (FDR).

To test differences in phylum and genus counts between immunizations and timepoints, the same model was used in the generalized linear model (GLM) implemented in the EdgeR package [31]. Pairwise comparisons of phylum and genus counts between timepoints and immunizations have been assessed with Fisher's exact test in EdgeR package.

Correlations of either the taxonomy counts (phylum and genus relative abundances) or the microbial counts from the traditional culture approach and disease features, such as anti-TSHR antibodies and thyroid hormone thyroxine levels (fT4), orbital adipogenesis, or muscular atrophy values, were estimated using the Spearman correlation coefficient (Rho) and represented in a correlation plot, using the $\mathrm{R}$ Corrplot package. Additional statistical methods are described in Additional file 2.

\section{Results}

\section{Sequencing metrics}

From 16S rRNA gene sequencing (V1-V2 regions), a total of $5,333,798$ reads were obtained which reduced to 4,047,186 reads after quality filtering. Following alignment, we obtained an average of 20,534 reads per sample, ranging from 3502 to 134,901 . Subsampling per library size resulted in a $96 \%$ average coverage per OTU definition at 3052 reads per sample. The averaged coverage and subsampling was sufficient to describe gut bacterial communities according to sequence-based rarefaction curves (data not shown).

We identified a total of 4281 OTUs: 1037 OTUs had more than 10 counts across samples and were retained.

\section{Comparative analysis of the gut microbiota of GO preclinical mouse models in different centers}

To assess whether the microbiota has an impact on the GO mouse model in different laboratories, we compared the gut microbial contents of 5 TSHR mice from Center 1 and 10 TSHR-immunized BALB/c female mice from Center 2, after sacrifice (T4).

Comparison of the alpha diversity indices showed a significant reduction in the richness $(P=0.01)$, but not in the diversity of the Center 2 microbial community $(P>0.05$, Fig. 1a). The gut microbiota composition from the two centers showed a good separation according to the Spearman distance and Ward hierarchical clustering (Fig. 1b), and a PERMANOVA test on the weighted Unifrac distances revealed a spatial difference between bacterial communities $(P=0.005$ with 999 permutations, Fig. 1c).

At a phylum level, Bacteroidetes and Firmicutes were the most represented of the seven phyla identified, with no differences between them in the two centers $(P=0.99)$. Lactobacillaceae, Ruminococcaceae, and Porphyromonadaceae families were more abundant in Center 2 than in Center 1 TSHR mice $(P<0.01$, Fig. $1 d)$. We observed significant differences in the abundance of 18 genera between the two centers, as detailed in Table 2 .

From the traditional microbial culture of the gut contents, we observed differences in yeast $(P=0.03186), \mathrm{Bac}$ teroides $(P<0.0005)$, and total anaerobe $(P=0.00081)$ counts, which were found to be enriched in the Center 1 compared with the Center 2 TSHR mice (Table 3). Cultures from mouse intestinal scraping of Center 2 did not contain any total clostridia, Bacteroides, or yeasts, and we were able to culture enterobacteria, E. coli, and coliforms from only one mouse from this group. E.coli and coliforms were also the least abundant in the Center 2 TSHR mice (Fig. 1e). Furthermore, since Yersinia enterocolitica has been implicated in GD pathogenesis [32], we used selective agar plates for Yersinia sp. but no Yersinia colonies grew.

\section{Gut microbiota differences in immunized and control mice within Center 2}

To observe the possible contribution of the gut microbiota in the disease, we compared the gut microbiota composition between immunization groups in mice within Center 2. No significant differences were observed in alpha diversity indices among immunizations, apart from the abundance-based coverage estimator (ACE) index between untreated and TSHR groups (Fig. 2a, $P=0.01$ ). The ACE index relies on the presence of rare OTUs [33], which were more abundant in the untreated group compared to the plasmid-immunized mice. The Bgal group showed a slightly skewed distribution of the Shannon index when compared to the others; however, the post hoc comparison was not significant.

The non-metric dimensional scaling (NMDS) of the weighted Unifrac distance matrix showed a separation of the three immunization groups, also confirmed by the permutation test $(P<0.01,999$ permutations; Fig. $2 b)$. 


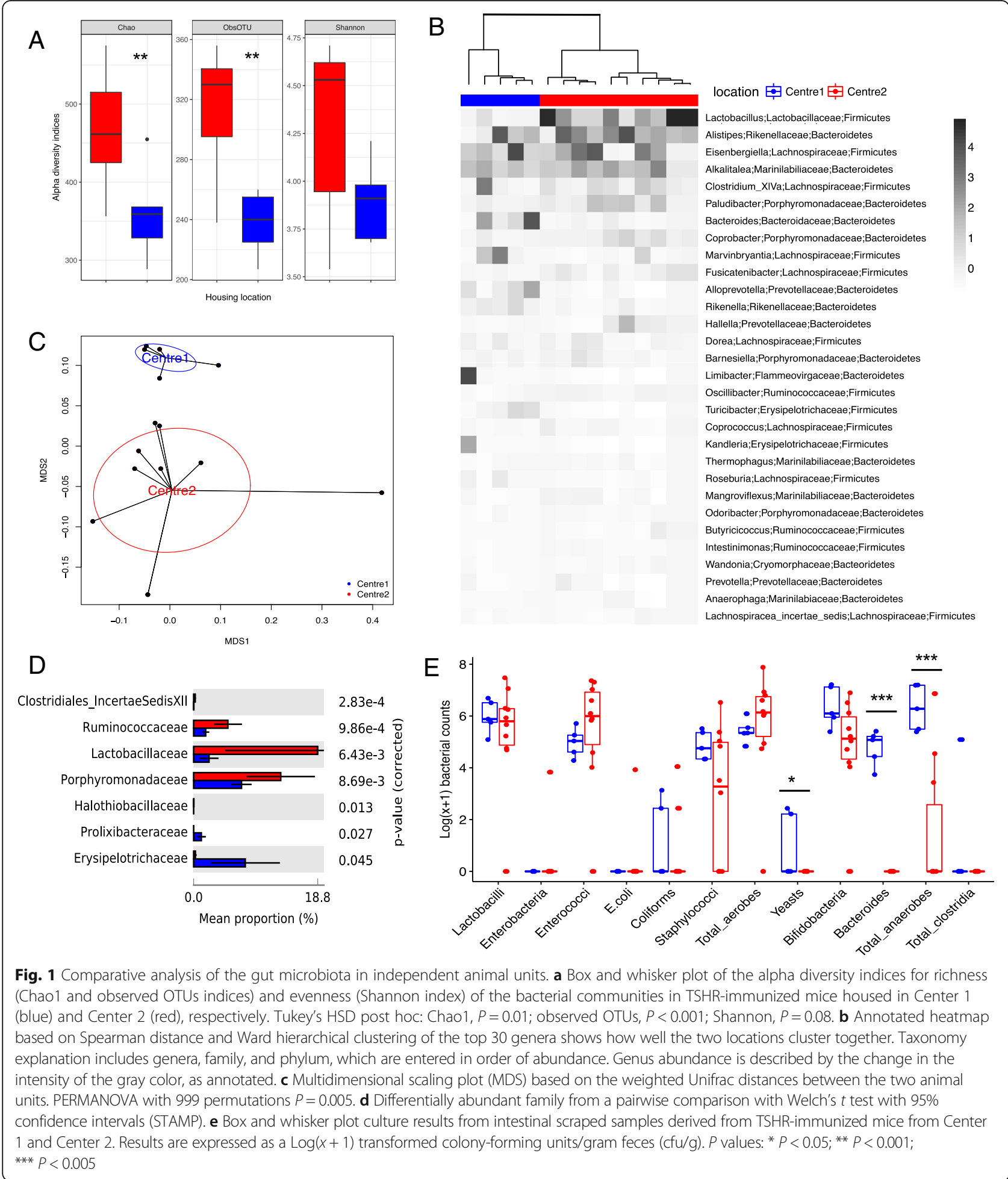

Bgal bacterial communities were closer to those of the untreated mice, while we observed a spatial shift of the TSHR-immunized bacterial communities.

OTUs from Bacteroidetes and Firmicutes phyla were the most abundant among the phyla identified (Fig. 2c) and showed a different distribution pattern among immunized groups. In particular, Firmicutes counts were higher in TSHR-immunized mice $(P=0.05)$ and Bacteroidetes were found to be higher in the untreated group $(P=0.012)$. At a genus level, eight genera were 
Table 2 Genera differentially abundant between Center $1(n=5)$ and Center $2(n=10)$ TSHR-immunized mice intestinal scraped samples

\begin{tabular}{llll}
\hline Genera & Center 1: mean freq. (\%) & Center 2: mean freq. (\%) & $P$ values \\
\hline Allobaculum & 1.001 & 0.003 & 0.042 \\
Alloprevotella & 6.135 & 0.432 & 0.003 \\
Bacteroides & 9.370 & 1.525 & 0.017 \\
Bifidobacterium & 0.668 & 0.006 & 0.003 \\
Clostridium XI & 0.840 & 0.000 & 0.005 \\
Coprobacter & 1.835 & 4.226 & 0.033 \\
Fusicatenibacter & 0.989 & 3.295 & 0.032 \\
Guggenheimella & 0.006 & 0.169 & 0.011 \\
Helicobacter & 0.200 & 0.000 & 0.024 \\
Intestinimonas & 0.097 & 0.861 & 0.000 \\
Lactobacillus & 2.304 & 18.632 & 0.030 \\
Lactonifactor & 0.023 & 0.401 & 0.025 \\
Meniscus & 1.149 & 0.000 & 0.000 \\
Oscillibacter & 0.640 & 1.748 & 0.011 \\
Parabacteroides & 0.292 & 0.031 & 0.015 \\
Pseudoflavonifractor & 0.154 & 0.466 & 0.028 \\
Rikenella & 3.921 & 1.216 & 0.004 \\
Turicibacter & 3.629 & 0.000 & 0.002 \\
\hline ANOvA with & 3.629 &
\end{tabular}

ANOVA with Tukey's HSD post hoc analysis (95\% confidence interval), generated with STAMP. Mean freq: mean frequency (\%)

differentially abundant between TSHR and ßgal groups, three genera between TSHR and the untreated group, and four genera between $\beta$ gal and the untreated group (Additional file 3: Table S1). We found an enrichment of OTUs of Acetitomaculum genus in the Bgal group compared to both TSHR $(P=0.004)$ and the untreated group $(P=0.003)$; an enrichment of Lactobacillus OTUs in the
TSHR compared to the untreated group $(P=0.018)$, and a reduction of Bacteroides counts in TSHR when compared to the $\beta$ gal group $(P=0.047)$. However, no significant differences were observed among immunized groups and in pairwise comparisons generated by the traditional bacterial culturing approach (Additional file 3: Table S2).

Table 3 Traditional microbiology results from TSHR-immunized mouse intestinal scraping from Center $1(n=5)$ and Center $2(n=10)$

\begin{tabular}{llll}
\hline Microbial target & Center 1: mean counts & Center 2: mean counts & $P$ values \\
\hline Bacteroides & $1.15 \mathrm{E}+05$ & b.d.l. & 0.000 \\
Bifidobacteria & $6.41 \mathrm{E}+06$ & $1.32 \mathrm{E}+06$ & 0.057 \\
Coliforms & $3.27 \mathrm{E}+02$ & $1.15 \mathrm{E}+03$ & 0.453 \\
E.coli & b.d.l. & $8.45 \mathrm{E}+02$ & 0.499 \\
Enterobacteria & b.d.l. & $6.82 \mathrm{E}+02$ & 0.499 \\
Enterococci & $1.74 \mathrm{E}+05$ & $6.10 \mathrm{E}+06$ & 0.247 \\
Lactobacilli & $1.93 \mathrm{E}+06$ & $4.68 \mathrm{E}+06$ & 0.725 \\
Staphylococci & $1.31 \mathrm{E}+05$ & $3.77 \mathrm{E}+05$ & 0.175 \\
Total aerobes & $4.18 \mathrm{E}+05$ & $9.90 \mathrm{E}+06$ & 0.370 \\
Total anaerobes & $6.75 \mathrm{E}+06$ & $7.39 \mathrm{E}+05$ & 0.001 \\
Total Clostridia & $2.46 \mathrm{E}+04$ & b.d.l. & 0.165 \\
Yeast & $8.72 \mathrm{E}+01$ & b.d.l. & 0.031 \\
\hline
\end{tabular}

b.d.I.: below detection limit. Detection limits are the following according to the agar used: $1000 \mathrm{CFU} / \mathrm{g}$ feces for Bacteroides, $100 \mathrm{CFU} / \mathrm{g}$ feces for E.coli and coliforms as well as for enterobacteria, and $10 \mathrm{CFU} / \mathrm{g}$ feces for total clostridia and yeasts, respectively. Microbiological counts were Box-Cox transformed. $P$ values obtained by linear regression 


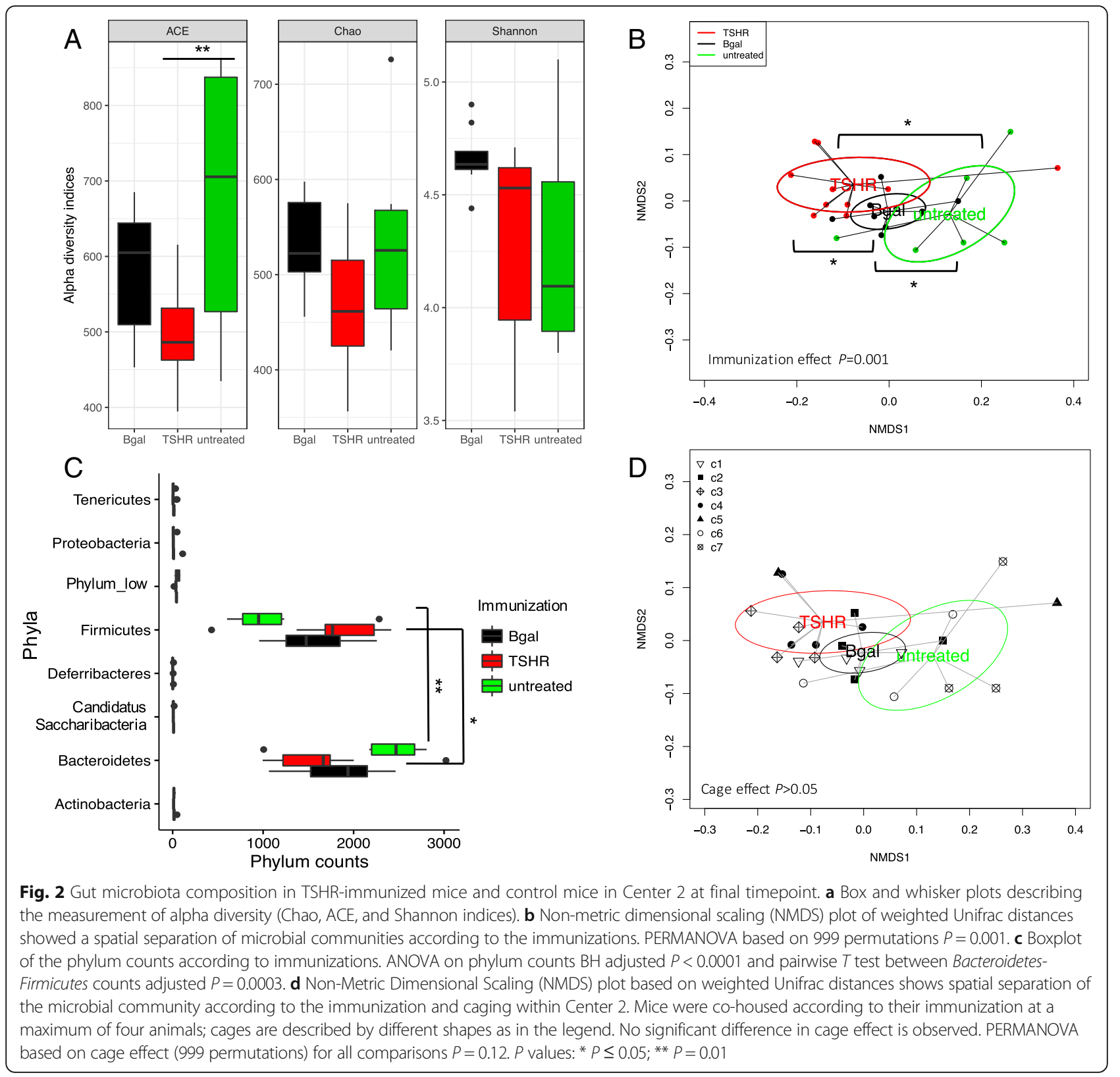

In the scraped intestinal samples, we did not observe a cage effect on the composition of the large intestine microbiota (PERMANOVA $P>0.05$; Fig. 2d).

\section{Dynamics of fecal microbiota during the immunization protocol}

To assess whether the immunization plasmids and the duration of the protocol could have influenced the gut microbiota composition, we sequenced the bacterial $16 \mathrm{~S}$ rRNA gene from the fecal pellets of the $\beta$ gal and TSHR group from the baseline (T0) for 18 weeks afterwards, until the end of the experiment (T4).

We observed a significant increase of the richness (Chao index, Fig. 3a; $P=0.02$ ) and the diversity
(Shannon index, Fig. 3b) over time, which was less apparent in the TSHR-immunized group. Significant differences regarding richness between TSHR and $\beta$ gal have been observed at T4 $(P=0.027$, Table 4$)$. The Shannon index of diversity, on the contrary, was significantly different between TSHR and Bgal immunization at T1 $(P=0.023$, Table 4).

The murine fecal microbiota comprised Bacteroidetes and Firmicutes phyla predominantly (Fig. 4c); followed by Tenericutes, Proteobacteria Deferribacteres, and Candidatus Saccharibacteria phyla. The Firmicutes/Bacteroidetes ratio has been used to describe the shift in the gut microbiota associated with aging [34] and also in disease conditions such as obesity [35]. The ratio showed 


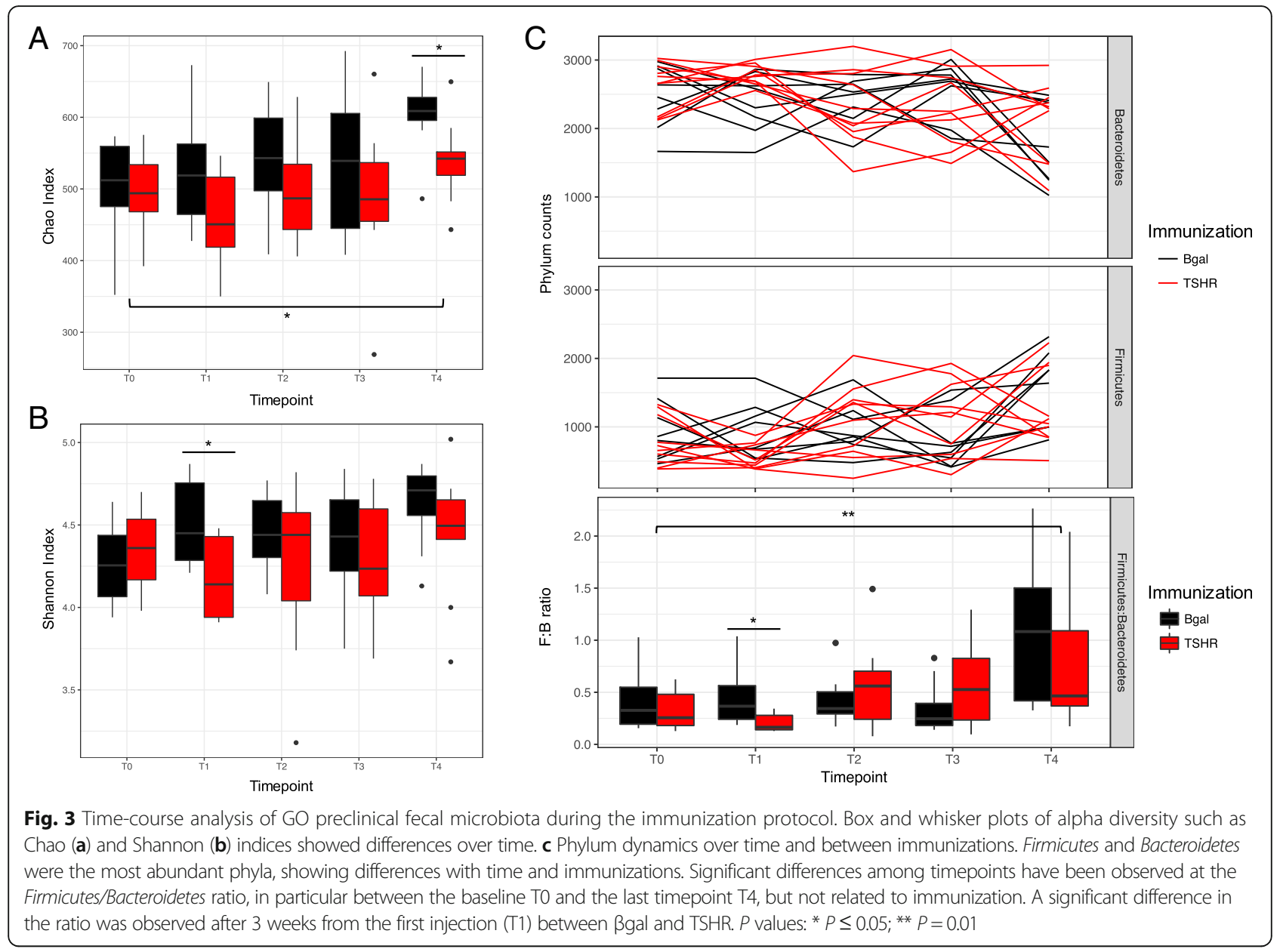

differences among the timepoints of the experimental procedure $(P<0.01)$ and between TSHR and the Bgal group after 3 weeks from the first injection (T1, $P=0.011$; Fig. 3c).

We fitted a generalized linear model (GLM) to compare the taxonomic counts at different timepoints within each group independently (either TSHR or $\beta$ gal). Thirty-four genera have been identified as differentially abundant among all timepoints in the TSHR-immunized group (Additional file 4: Table S1), while 25 were found in the $\beta$ gal group (Additional file 4: Table S2). We observed differences in the taxonomic profile between TSHR and $\beta$ gal groups at each timepoint using an exact test (EdgeR). Once again, T1 was identified as the timepoint with the highest number of genera differentially expressed, as illustrated by the diversity indices (Additional file 4: Table S3).

In contrast to data obtained from the gut microbiota (Fig. 2d), a cage effect was observed in the fecal microbiota, in particular, in interaction with time $(P=0.001)$ and immunization $(P=0.002$; Additional file 5: Figure $\mathrm{S} 1)$. The latter is probably due to the mice being caged according to the type of plasmid injection they received, but we also observed a significant difference within the same immunization group (e.g., TSHR in cage 4 and cage $5, P=0.01$ ).

Table 4 Summary of the statistics from the time-course analysis of the fecal microbiota during the immunization protocol (T0-T4) and between immunizations ( $\beta g a l$ and TSHR)

\begin{tabular}{|c|c|c|c|c|c|c|c|c|}
\hline \multirow[t]{2}{*}{ Index } & \multicolumn{3}{|l|}{ ANOVA model } & \multicolumn{5}{|c|}{ TSHR vs. ßgal group } \\
\hline & Immunization & Time & Time $\times$ immunization & T0 & $\mathrm{T} 1$ & $\mathrm{~T} 2$ & T3 & T4 \\
\hline Chao & 0.006 & 0.02 & 0.8 & 0.75 & 0.066 & 0.28 & 0.33 & 0.027 \\
\hline Shannon & 0.054 & 0.28 & 0.47 & 0.44 & 0.023 & 0.35 & 0.35 & 0.29 \\
\hline Firm:Bact & 0.406 & 0.0003 & 0.16 & 0.39 & 0.028 & 0.46 & 0.2 & 0.26 \\
\hline
\end{tabular}

Firm:Bact, Firmicutes/Bacteroidetes ratio. ANOVA model as previously described. Pairwise comparison between $\beta$ gal and TSHR in each time point has been made with a pairwise T-test with Benjamini-Hochberg correction for FDR 

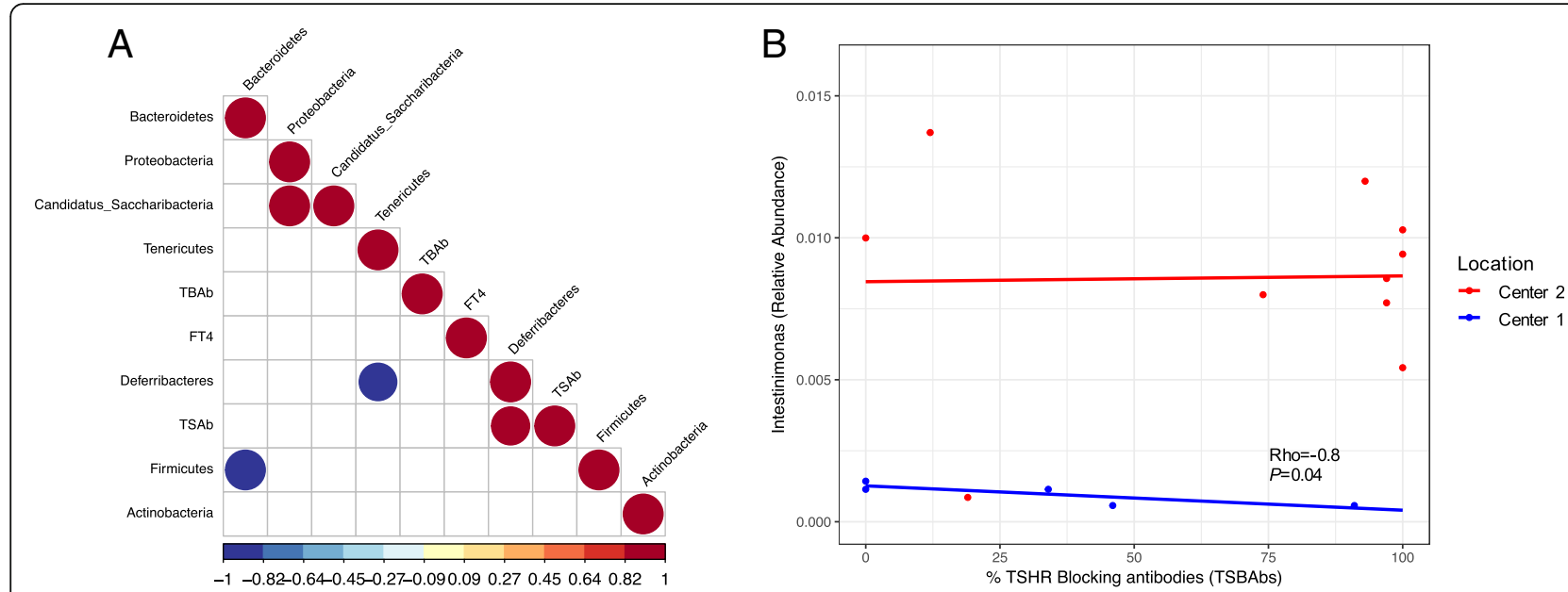

Fig. 4 Correlating the gut microbiota and disease features in Center 2 TSHR group. a Spearman correlation coefficient strength (Rho) of phylum counts from TSHR mice in Center 2. Firmicutes and Bacteoridetes showed a strong negative correlation between each other. A positive correlation between the one-genus phylum Deferribacteres and the level of thyroid-stimulating antibodies (TSAb) has been observed. Correlations with $P<$ 0.05 are shown and strength of the Rho coefficient is represented by the change in the color intensity. fT4, free thyroid hormone thyroxine levels; TSAb, thyroid stimulating antibodies; TSBAb, thyroid-stimulating blocking antibodies (as a percentage values). $\mathbf{b}$ Enriched Firmicutes genus Intestinimonas between Center 1 (blue) and Center 2 (red) showed a strong negative correlation with the percentage of thyroid-stimulating blocking antibodies (TSBAbs) at 95\% confidence interval in Center 1 (Rho $=-0.8, P=0.04$ ), but not in Center 2

\section{Correlating the gut microbiota composition with clinical features and differences in GO development}

We then investigated possible correlations between disease features, such as anti-TSHR antibodies, thyroxine levels (fT4), orbital adipogenesis, and muscular atrophy, and the gut microbiota composition to determine whether it contributes to the heterogeneity of induced responses, summarized in Additional file 1: Table S1.

Within the Center 1 TSHR-immunized group, we found that OTUs from Firmicutes and Bacteroidetes negatively correlated to each other (Rho $=-1, P<0.0001$ ). A positive correlation between levels of TSAb and Deferribacteres phylum, which include one-genus Mucispirillum, was found (Rho $=0.92, P=0.028$; Fig. 4a).

From those genera differentially abundant between TSHR-immunized mice from Center 1 and Center 2 (Table 2), identified via metataxonomics, we observed a strong negative correlation of the Firmicutes genus Intestinimonas and the levels of TSBAb in the Center 1 (Rho $=-0.89, P<0.05$ ) but not in the Center 2 counterpart (Fig. 4b). No significant correlation was observed between OTUs from the genus Intestinimonas and levels of TSAb or levels of free thyroxine hormone (fT4; data not shown).

On the contrary, the Box-Cox transformed counts from the traditional microbiology did not show any significant correlation with the disease features described (data not shown).

Within Center 2, Bacteroidetes and Firmicutes negatively correlated to each other $($ Rho $=-0.99, P<0.0001$;
Fig. 5a). We also found a significant positive correlation (Rho $=0.6, P=0.009$ ) between the OTUs from the Firmicutes and the orbital adipogenesis value and a negative correlation of this value with the phylum Bacteroidetes (Rho $=-0.57, P=0.014$ ). As expected, these correlations were specific to the TSHR-immunized mice (Fig. 5b). The correlation pattern we found (Firmicutes positively correlated, Bacteroidetes negatively correlated) was also recapitulated at the genus level. Among the genera of the Firmicutes, three within the Clostridia family (Butyricicoccus, Parvimonas and Fusibacter) and the genus Lactobacillus were correlated positively with adipogenesis, while three Bacteroidetes genera (Anaerophaga, Paraprevotella, and Tannerella) correlated negatively with the orbital adipogenesis values (Fig. 5c).

A strong positive correlation $($ Rho $=0.82, P=0.007$ ) was observed between orbital adipogenesis and the total anaerobes counts obtained from the traditional microbial cultures of TSHR-immunized mice, but not in the controls (Fig. 5d). Moreover, from the traditional microbial cultures data, we observed correlations with other disease features, specifically in the TSHR group. We observed strong positive correlations between the muscular atrophy values and the cluster of lactobacilli (Rho $=0.74, P=0.03$ ), enterococci (Rho $=0.8, P=0.02$ ), bifidobacteria (Rho $=0.76$, $P=0.03$ ), and coliforms (Rho $=0.73, P=0.04)$. Levels of free thyroxine (fT4) were positively correlated with lactobacilli (Rho $=0.64, P=0.05)$ and staphylococci (Rho $=0.77, P=0.016$ ). 


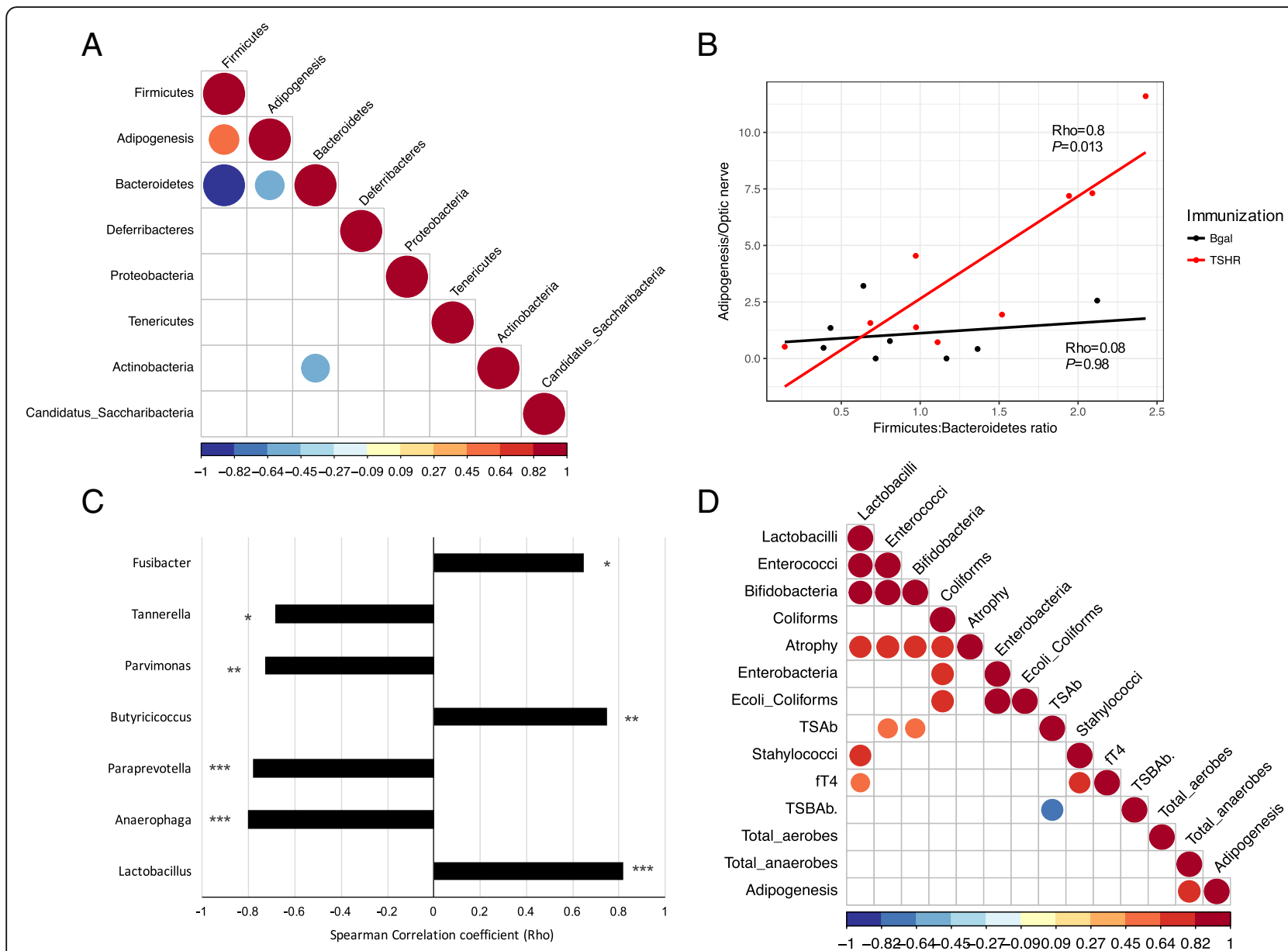

Fig. 5 Correlation of the gut microbiota composition with clinical features and differences in Center 2 mice. a Correlation plot of phyla and the orbital adipogenesis value. Spearman correlation coefficient strength (Rho) as indicated by the colored bar. Firmicutes and Bacteoridetes showed a strong negative correlation between each other. A positive correlation between Firmicutes and a negative correlation with Bacteroidetes OTUs and the adipogenesis value (calculated in the orbit) has been observed. Adipogenesis clustered closer to the Firmicutes and Bacteroidetes value according to the complete linkage method for hierarchical clustering. Only $P<0.05$ are shown. b Positive strong correlation of the Firmicutes/Bacteroidetes ratio with the adipogenesis value (calculated in the orbit) resulted significant in TSHR-immunized group but not in the ßgal group. c Spearman correlation coefficient (Rho) of genera among phyla Bacteroidetes and Firmicutes and the orbital adipogenesis values. The strength of the correlation coefficient is represented on $x$-axis: bars on the left represent a negative correlation coefficient, while bars on the right represent a positive correlation coefficient. Correlations with $P<0.05$ are shown; order of entrance depends on their $P$ values: ${ }^{*} P<0.05$; ${ }^{* *} P<0.1$; ${ }^{* *} P<0.005$. d Spearman correlation coefficient plot of the Box-Cox transformed microbiological counts and disease features in Center 2 TSHR-immunized mice. Feature clustering was according to the complete linkage method for hierarchical clustering. Only correlations with $P<0.05$ are shown and strength of the correlation coefficient is represented by the change in the color intensity. fT4, free thyroid hormone thyroxine levels; TSAb, thyroid-stimulating antibodies; TSBAb, thyroid-stimulating blocking antibodies (as a percentage values)

\section{Discussion}

Animal models have been invaluable in dissecting the mechanisms causing loss of immune tolerance leading to autoimmune conditions such as GD. Thus, we aimed to test the hypothesis that the gut microbiota may affect both outcome and reproducibility of induced autoimmune disease, such as reported in the recent research article of Berchner-Pfannschmidt and co-workers [12].

We observed significant differences in the diversity and spatial organization of the gut microbiota of female TSHR-immunized BALBc mice in two independent SPF units. We also demonstrated disease-associated microbial taxonomies and correlation with ocular disease, suggesting that the gut microbiota have contributed to the heterogeneity of induced response in the two locations, which further supports our hypothesis.

Animals were maintained in similar conditions. We are confident that there were no infections ongoing at the moment of sampling, since animals in both centers were routinely tested for the presence of viruses, mycoplasma and parasites (see Additional file 1: Table S2); moreover, housing facilities had comparable SPF conditions. Animals were from the same supplier but in different countries (Harlan Ltd. for Center 1 and Harlan Lab. 
BV for Center 2), had received autoclaved water, and had been fed similar commercial chow, with the exception that food pellets provided in Center 2 contained twice the amount of iodide compared to Center 1 food (see Additional file 1: Table S3). Although iodide excess can be associated with abnormal thyroid function, we do not consider that this dietary variation is enough to explain the results (i.e., elevated thyroxine levels were apparent in the Center 1 but not in the Center 2 mice). The effect of iodine has been studied in the NOD mouse which spontaneously develops autoimmune thyroiditis. Vecchiatti and colleagues [36] reported that excess iodine $(0.2 \mathrm{mg} / \mathrm{mouse} /$ day $)$ increased the incidence and severity of disease; however, the BALB/c mice in our study did not display thyroiditis. A transgenic NOD mouse expressing the human TSHR-A subunit is able to develop antibodies to the human TSHR and this too is exacerbated by iodine excess [37] but at levels far greater than in the chow used in Centers 1 and 2. We also considered whether iodine could affect the gut microbiota, in view of its use as an antiseptic, but all the studies we found were in this context, rather than the effect of dietary iodine on symbionts. The importance of SPF conditions is indicated by a previous study which failed to reproduce a GO animal model, despite using mice from the same supplier and identical bedding, water, and chow [10]. However, even SPF may be inadequate since differences were found in the gut microbiota of C57BL/6 colonies bred in two different rooms of the same SPF facility [38], fortunately mice in our study were all housed in the same room.

Cage effects were apparent in the fecal microbiota results, which highlight the importance of studying the gut microbiota instead when comparing autoantigen (TSHR)-immunized and control mice, which are in the close proximity of the intestinal mucosa and the immune system, enabling us to explore its relationship with disease features.

We observed several disease-associated taxonomies; the abundance of the newly described butyrate-producing genus Intestinimonas [39] was reduced in the Center 1 group compared to Center 2 and correlated negatively with TSBAb. The Intestinimonas species butyroproducens has a unique ability to produce butyrate from lysine and is involved in the detoxification of advanced glycosylation end (AGE) products such as fructoselysin, which have been linked to type 1 diabetes [40], although we are unaware of any link between butyrate-producing bacteria and thyroid autoimmunity.

The TSHR-immunized group developed some signs of GO and their gut microbiota had increased OTUs of the phylum Firmicutes but decreased Bacteroidetes compared with controls. This mirrors our preliminary data in human disease where we observed a dramatic reduction in the Bacteroides genus in GD patients when they develop GO (INDIGO publishable summary ${ }^{1}$ ).

We also obtained a positive correlation between several Firmicutes counts, such as Clostridia and Bacilli, with orbital adipogenesis in Center 2 TSHR-immunized mice. Million and co-workers have previously reported a positive correlation between OTUs from the Firmicutes and weight gain/obesity in both animal models and humans [41]. Interestingly, the role of the genus Lactobacillus and its products in either triggering or protecting from adipogenesis has been debated and seems to be species-specific.

In the present work, we could exclude a possible gain-of-weight relationship with the adipogenesis value calculated in the orbit since no changes in mouse weights have been observed during the development of the chronic phase of the disease (data not shown). Furthermore, molecular mechanisms driving obesity and orbital adipogenesis may well be different, since the latter is derived from the neural crest and the gut microbiota may have varying effects on different fat depots [42].

Our time-course analysis revealed that time had a dramatic role in shaping the fecal microbiota of the female mice which were 6-8 weeks old at the outset and 24-26 weeks at the end of the experiment, confirming the work of McCafferty and colleagues [43]. The richness and diversity of $\beta$ gal control mice increased with age but this was less apparent in TSHR-immunized animals. Significant differences in microbiota composition between control and TSHR immunizations were most apparent 3 weeks after the first immunization, at the initiation of the induced immune response.

Our control group comprised mice immunized with the Bgal expression plasmid in which we observed a slight skew in the microbiota richness and diversity which may be caused by the systemic overexpression of the $\beta$-galactosidase enzyme, whose natural role is in glycan metabolism, e.g., the hydrolysis of the lactose to galactose and glucose [44]. Kaneda and collaborators reported a $\beta$ gal overexpression peak in the muscle fibers following electroporation from 5 days to 2 weeks after the injection [45].

It may be that the increased OTUs of the Firmicutes genus Acetitomaculum was specifically triggered by the product of the $\beta$ gal enzymatic reaction over time (Additional file 4: Table S2). This effect merits further investigation but we are confident that the $\beta$ gal vector plasmid provides the optimum control group since its microbial communities were more closely related to that of the naïve non-immunized group than to TSHR-immunized mice. Of interest, TSHR-immunized mice in Center 2 were more similar to TSHR-immunized mice from Center $1(P=0.2)$ than 
Bgal $(P=0.024)$, than untreated $(P=0.04)$ mice in their own center (Additional file 6: Figure S1).

The results we obtained using $16 \mathrm{~S}$ rRNA gene metataxonomics and via the traditional microbial culture approach were largely similar, with relatively few differences. Microbial cultures revealed significantly higher yeast counts $(P=0.03186)$ in Center 2 TSHR-immunized mice-which obviously could not be seen via the bacterial metataxonomics - and a nearly significant difference in the Actinobacteria genus Bifidobacterium $(P=0.057)$, which was not detected in our metataxonomics data. Primers based on the V1-V2 regions of the 16S rRNA gene did not detect Bifidobacterium OTUs. Consequently, we applied a new set of primers (28F-combo) with which we observed a significant enrichment of bifidobacteria counts in Center 2 (Additional file 7: Figure S1), in agreement with the microbial culture results.

\section{Conclusions}

In conclusion, our results indicate a role for the gut microbiota in modulating the heterogeneity apparent in the TSHR-induced model of GD and GO. In our next study, we will report the effects on our induced model of modifying the gut microbiota using antibiotics, probiotics, and fecal material transfer.

Our future studies will investigate whether the presence, absence, or amounts of certain bacteria or yeast have the ability to directly alter the immune balance between the Treg anti-inflammatory response and the Th17-mediated pro-inflammatory response in the gut mucosa as has been reported in models of other autoimmune diseases [22, 46]. Results of these experiments could then be confirmed by colonization studies in gnotobiotic animals. Factors such as level of dietary iodine intake and age of mice at immunization, which may both alter the gut microbiota and/or immune responsiveness, are also warranted.

\section{Endnotes}

${ }^{1}$ INDIGO publishable summary: http://www.indigo-iapp.eu/publishable-summary/

\section{Additional files}

Additional file 1: Figure S1. Schematic representation of the GO immunization protocol and sample collection. Table S1. Summary of disease characteristics induced in mice in Center 1 and Center 2 using TSHR expression plasmid illustrating the heterogeneity of response. Table S2. Quarterly Health Screen Reports on viral, bacterial, mycoplasma and parasite screen in both centers. Table $\mathbf{S 3}$. Composition of the commercial chows provided ad libitum in Center 1 and Center 2. (DOCX $106 \mathrm{~kb})$

Additional file 2: Supplementary methods. (DOCX $121 \mathrm{~kb}$ )
Additional file 3: Table S1. Differential abundant taxonomic analysis between TSHR $(n=10)$, ßgal $(n=8)$, and untreated $(n=6)$, within Center 2. Welch's T-test with 95\% confidence interval using STAMP. Mean relative frequency, rel. freq. Standard deviation, std. dev. Table S2. Comparison of intestinal scraped samples from different immunization within Center 2 from the traditional microbiological culture. Data were Box-Cox transformed. (XLSX $43 \mathrm{~kb}$ )

Additional file 4: Table S1. Generalized linear model (GLM) of genera counts differentially present in TSHR-immunized mice over timepoints, in reference to the baseline (TO) using EdgeR. LogFC, Log2 fold change between each timepoint and the baseline (T0); LR, likelihood ratio. Table S2. Generalized linear model (GLM) of genera counts in $\beta$ gal control mice over timepoints using EdgeR. LogFC, Log2 fold change between each timepoint and the baseline (T0); LR, likelihood ratio. Table S3. Pairwise comparison of TSHR and $\beta g a l$ mice using Fisher's Exact Test in EdgeR at each timepoint (T0 to T4). LogFC, Log2 fold change of $\beta$ gal compared to TSHR. (XLSX 46 kb)

Additional file 5: Figure S1. Temporal stability of fecal microbiota and cage effect of the immunizations. Weighted Unifrac distances of mice fecal microbial communities represented over the time course of the experiment according to the immunization (A) or the cage (B). Permutational MANOVA of weighted Unifrac distances according to timepoint, immunizations, caging, and their interactions (time $\times$ cage: time $\times$ immunization; immunization $\times$ cage) as described in Additional file 2. The time had a significant effect on the stability of the fecal microbiota $(P=0.001)$, in particular between the baseline (T0) and the last timepoint $(\mathrm{T} 4, P=0.003)$; and between the $\mathrm{T} 1$ and $\mathrm{T} 4(P=0.009)$. The interaction between time and immunization was significant $(P=$ 0.007). Cage was also significant, in particular the interaction cage $x$ timepoint $(P=0.001)$ and cage $\times$ immunization $(P=0.002)$. Significant differences within the same immunization group cage has been observed (TSHR group in C4 and C5, P=0.01). (PDF $152 \mathrm{~kb}$ )

Additional file 6: Figure S1. NMDS plot based on the weighted Unifrac distances of Center2 immune and control mice including TSHRimmunized mice from Center 1. TSHR-immunized mice from Center 1 were more similar to TSHR-immunized mice from Center $2(P=0.2)$ than to the $\beta$ gal $(P=0.024)$ than the untreated $(P=0.04)$. (PDF $28 \mathrm{~kb})$

Additional file 7: Figure S1. Bifidobacterium counts derived from the 28F-combo primers in the TSHR-immunized mice in Center $1(n=5)$ and Center $2(n=10)$. ANOVA with Tukey's HSD post hoc analysis ( $95 \%$ confidence interval), $P$ value $=0.003$ generated with STAMP. (PDF $21 \mathrm{~kb}$ )

\section{Abbreviations}

ACE: Abundance-based coverage estimator; AGE: Advanced glycosylation end; CNS: Central nervous system; EAE: Experimental autoimmune encephalomyelitis; FDR: False discovery rate; fT4: Thyroid hormone thyroxine; GD: Graves' disease; GLM: Generalized linear model; GO: Graves' orbitopathy or ophthalmopathy; HSD: Honest significant difference; NMDS: Non-metric dimensional scaling; OTU: Operational taxonomic unit;

PERMANOVA: Permutational multivariate analysis of variance; RDP: Ribosomal Database Project; SFB: Segmented filamentous bacteria; SPF: Specificpathogen-free; TRAB: Thyroid-stimulating hormone autoantibodies; Treg: Regulatory T cells; TSAb: Thyroid-stimulating antibodies; TSBAb: Thyroid-stimulating blocking antibodies; TSH: Thyroid-stimulating hormone; TSHR: Thyrotropin receptor; $\beta$ gal: $\beta$-Galactosidase enzyme

\section{Acknowledgements}

We acknowledge the work of the other members of the INDIGO consortium: Mario Salvi, Lei Zhang, Giuseppe Colucci, Daryn Michael, and Iveta Garaiova. We also acknowledge the invaluable help of Dr. Ann Smith.

\section{Funding}

Supported by Marie-Sklodowska Curie Industry Industry-Academia Pathways and Partnerships (IAPP) action, GA number 612116 project INDIGO. Deutsche Forschungsgemeinschaft grant BE3177/2-1 (UBP). Internal KCL funds (JPB). 


\section{Availability of data and materials}

The datasets used and/or analyzed during the current study are available from the corresponding author on reasonable request, since we are waiting for the repository accession number.

\section{Authors' contributions}

SM collected the data and was involved in writing the report with GM and HLK. GM analyzed the data with FB and JRM. HLK, DC, JPB, UBP, MH, SDC, GEG, SP, and AE contributed to the study design and sample collection. ML designed and managed the project, supervised the analyses, and contributed to the report. All authors read and agreed to the final version of the manuscript.

\section{Ethics approval and consent to participate}

The study was approved by the North Rhine Westphalian State Agency for Nature, Environment and Consumer Protection, Germany and by the Ethics Committee of King's College London, United Kingdom (UK).

\section{Competing interests}

The authors declare that they have no competing interests.

\section{Publisher's Note}

Springer Nature remains neutral with regard to jurisdictional claims in published maps and institutional affiliations.

\section{Author details \\ 'Division of Infection \& Immunity, School of Medicine, Cardiff University, UHW main building, Heath Park, Cardiff CF14 4XW, UK. ${ }^{2}$ Departments of Bioinformatics, PTP Science Park Srl, via Einstein loc. Cascina Codazza, 29600 Lodi, Italy. ${ }^{3}$ Molecular Ophthalmology, Department of Ophthalmology, University Hospital Essen/University of Duisburg-Essen, 45147 Essen, Germany. ${ }^{4}$ Faculty of Life Sciences and Medicine, King's College London, London SE5 9NU, UK. ${ }^{5}$ Latner Thoracic Surgery Laboratories, Toronto General Research Institute, University Health Network and University of Toronto, Toronto M5G 1L7, Canada. ${ }^{6}$ Cultech Ltd., Baglan, Port Talbot SA127BZ, UK. 'University Hospital Essen, University of Duisburg-Essen, Institute of Medical Microbiology, 45147 Essen, Germany. ${ }^{8}$ Graves' Orbitopathy Center, Endocrinology, Department of Clinical Sciences and Community Health, Fondazione Ca'Granda IRCCS, University of Milan, via Sforza 35, 20122 Milan Italy. ${ }^{9}$ King's College Hospital NHS Foundation Trust (SDC), London SE5 9RS, UK. ${ }^{10}$ Italian National Council for Research (CNR), via Bassini 15, 20133 Milan, Italy. ${ }^{11}$ School of Biosciences, Cardiff University, Sir Martin Evans Building, Museum Avenue, Cardiff CF10 3AX, UK. ${ }^{12}$ Center for Digestive and Gut Health, Imperial College London, W2 1NY, London, UK. ${ }^{13}$ INDIGO Consortiumhttp://www.indigo-iapp.eu.}

\section{Received: 18 October 2017 Accepted: 8 May 2018}

Published online: 25 May 2018

\section{References}

1. Ericsson AC, Davis JW, Spollen W, Bivens N, Givan S, et al. Effects of vendor and genetic background on the composition of the fecal microbiota of inbred mice. PLoS One. 2015;10:e0116704.

2. Hufeldt MR, Nielsen DS, Vogensen FK, Midtvedt T, Hansen AK. Variation in the gut microbiota of laboratory mice is related to both genetic and environmental factors. Compar Med. 2010;60:336-47.

3. Draman M, Ludgate M. Thyroid eye disease-an update. Exp Rev Ophthalmol. 2016;11:1-12.

4. McLachlan SM, Rapoport B. Breaking tolerance to thyroid antigens: changing concepts in thyroid autoimmunity. Endocr Rev. 2014;35:59-105.

5. Morshed SA, Davies TF. Graves' disease mechanisms: the role of stimulating, blocking and cleavage region TSHR receptor antibodies. Horm Metab Res. 2015;47(Suppl 10):727-34.

6. Bahn RS. Graves' ophthalmopathy. New Engl J Med. 2010;362:726-38.

7. Banga JP, Moshkelgosha S, Berchner-Pfannschmidt U, Eckstein A. Modelling Graves' orbitopathy in experimental Graves' disease. Horm Metab Res. 2015; doi: https://doi.org/10.1055/s-0035-1555956.

8. Ludgate M. Animal models of Graves' disease. Eur J Endocrinol. 2000; 142:1-8.
9. Many MC, Costagliola S, Detrait M, Denef JF, Vassart G, Ludgate M. Development of an animal model of autoimmune thyroid eye disease. J Immunol. 1999;162:4966-74.

10. Baker G, Mazziotti G, von Ruhland C, Ludgate M. Reevaluating thyrotropin receptor-induced mouse models of Graves' disease and ophthalmopathy. Endocrinology. 2005;146:835-44.

11. Bhattacharyya KK, Coenen MJ, Bahn RS. Effect of environmental pathogens on the TSHR-directed immune response in an animal model of Graves' disease. Thyroid 2005;15:422-6.

12. Berchner-Pfannschmidt U, Moshkelgosha S, Diaz-Cano S, Edelmann B, Görtz G-EE, Horstmann M, et al. Comparative assessment of female mouse model of Graves' orbitopathy under different environments, accompanied by proinflammatory cytokine and T cell responses to thyrotropin hormone receptor antigen. Endocrinology. 2016;157:1673-82.

13. Moshkelgosha S, So P-W, Deasy N, Diaz-Cano S, Banga J. Retrobulbar inflammation, adipogenesis, and acute orbital congestion in a preclinical female mouse model of Graves' orbitopathy induced by thyrotropin receptor plasmid-in vivo electroporation. Endocrinology. 2013;154:3008-15.

14. Murri M, Leiva I, Gomez-Zumaquero JM, Tinahones FJ, Cardona F, et al. Gut microbiota in children with type 1 diabetes differs from that in healthy children: a case-control study. BMC Med. 2013;11:46.

15. Brown $C T$, Davis-Richardson AG, Giongo A, Gano KA, Crabb DB, et al. Gut microbiome metagenomics analysis suggests a functional model for the development of autoimmunity for type 1 diabetes. PLoS One. 2011;6:25792.

16. Frank DN, St. Amand AL, Feldman RA, Boedeker EC, Harpaz N, Pace NR. Molecular-phylogenetic characterization of microbial community imbalances in human inflammatory bowel disease. Proc Natl Acad Sci U S A. 2007:104:13780-5.

17. Scanlan PD, Shanahan F, O'Mahony C, Marchesi JR. Culture-independent analyses of temporal variation of the dominant fecal microbiota and targeted bacterial subgroups in Crohn's disease. J Clin Microbiol. 2006;44: 3980-8.

18. Laukens D, Brinkman BM, Raes J, De Vos M, Vandenabeele P. Heterogeneity of the gut microbiome in mice: guidelines for optimizing experimental design. FEMS Microbiol Rev. 2016;40:117-32.

19. Ochoa-Repáraz J, Mielcarz DW, Ditrio LE, Burroughs AR, Foureau DM, et al. Role of gut commensal microflora in the development of experimental autoimmune encephalomyelitis. J Immunol. 2009;183:6041-50.

20. Lee YK, Menezes JS, Umesaki Y, Mazmanian SK. Proinflammatory T-cell responses to gut microbiota promote experimental autoimmune encephalomyelitis. Proc Natl Acad Sci U S A. 2011;108(Suppl 1):4615-22.

21. Covelli $\mathrm{D}$, Ludgate $\mathrm{M}$. The thyroid, the eyes and the gut: a possible connection. J Endocrinol Investig. 2017; https://doi.org/10.1007/s40618-016-0594-6.

22. Zhao SX, Tsui S, Cheung A, Douglas RS, Smith TJ, Banga JP. Orbital fibrosis in a mouse model of graves' disease induced by genetic immunization of thyrotropin receptor CDNA. J Endocrinol. 2011;210:369-77.

23. Box GEP, Cox DR. An analysis of transformations. J R Stat Soc B. 1964;26: $211-43$.

24. Schloss PD, Westcott SL, Ryabin T, Hall JR, Hartmann M, et al. Introducing mothur: open-source, platform-independent, community-supported software for describing and comparing microbial communities. Appl Environ Microbiol. 2009; https://doi.org/10.1128/AEM.01541-09.

25. Edgar RC, Haas BJ, Clemente JC, Quince C, Knight R. UCHIME improves sensitivity and speed of chimera detection. Bioinformatics. 2011;27: 2194-200.

26. Cole JR, Wang Q, Cardenas E, Fish J, Chai B, et al. The ribosomal database project: improved alignments and new tools for rRNA analysis. Nucl Acids Res. 2009;37:D141-5.

27. Price MN, Dehal PS, Arkin AP. FastTree 2-approximately maximumlikelihood trees for large alignments. PLoS One. 2010;5:e9490.

28. Parks DH, Tyson GW, Hugenholtz P, Beiko RG. STAMP: statistical analysis of taxonomic and functional profiles. Bioinformatics. 2014; https://doi.org/10. 1093/bioinformatics/btu494.

29. Lozupone C, Lladser ME, Knights D, Stombaugh J, Knight R. UniFrac: an effective distance metric for microbial community comparison. ISME J. 2011; 5:169-72.

30. Anderson MJ. A new method for non-parametric multivariate analysis of variance. Austral Ecol. 2001:26:32-46.

31. Robinson MD, McCarthy DJ, Smyth GK. edgeR: a Bioconductor package for differential expression analysis of digital gene expression data. Bioinformatics. 2010; https://doi.org/10.1093/bioinformatics/btp616. 
32. Köhling HL, Plummer SF, Marchesi JR, Davidge KS, Ludgate M. The microbiota and autoimmunity: their role in thyroid autoimmune diseases. Clin Immunol. 2017; https://doi.org/10.1016/j.clim.2017.07.001.

33. Chao A. Species estimation and application. In: Kotz S, Balakishnan N, et al., editors. Encyclopedia of statistical sciences. New York: Wiley; 2005. p. 7907-15.

34. Mariat D, Firmesse $\mathrm{O}$, Levenez F, Guimarăes VD, Sokol H, et al. The firmicutes/bacteroidetes ratio of the human microbiota changes with age. BMC Microbiol. 2009;9:123.

35. Ley RE, Turnbaugh PJ, Klein S, Gordon Jl. Microbial ecology: human gut microbes associated with obesity. Nature. 2006;444:1022-3.

36. Vecchiatti S, Guzzo M, Caldini E, Bisi H, Longatto-Filho A, et al. lodine increases and predicts incidence of thyroiditis in NOD mice: histopathological and ultrastructural study. Exp and therap med. 2013;5: 603-7.

37. Rapoport B, Aliesky HA, Banuelos B, Chen C-RR, McLachlan SM. A unique mouse strain that develops spontaneous, iodine-accelerated, pathogenic antibodies to the human thyrotrophin receptor. J Immunol. 2015:194:4154-61

38. Jakobsson H, Rodríguez-Piñeiro A, Schütte A, Ermund A, Boysen P, et al. The composition of the gut microbiota shapes the colon mucus barrier. EMBO Rep. 2015;16:164-77.

39. Kläring K, Hanske L, TPN B, Charrier C, Blaut M, et al. Intestinimonas butyriciproducens gen. nov., sp. nov., a butyrate-producing bacterium from the mouse intestine. Int J Syst Evol Microbiol. 2013; https://doi.org/10.1099/ ijs.0.051441-0.

40. Bui TPN, Ritari J, Boeren S, de Waard P, Plugge CM, Vos WM. Production of butyrate from lysine and the Amadori product fructoselysine by a human gut commensal. Nat Commun. 2015;6:10062.

41. Million M, Lagier JC, Yahav D, Paul M. Gut bacterial microbiota and obesity. Clin Microb Inf. 2013;19:305-13.

42. Anderson DJ, Axel R. Molecular probes for the development and plasticity of neural crest derivatives. Cell. 1985;42(2):649-62.

43. McCafferty J, Mühlbauer M, Gharaibeh R, Arthur J, Perez-Chanona E, et al. Stochastic changes over time and not founder effects drive cage effects in microbial community assembly in a mouse model. ISME J. 2013;7:2116-25.

44. Juers DH, Matthews BW, Huber RE. LacZ galactosidase: structure and function of an enzyme of historical and molecular biological importance. Protein Sci. 2012;21:1792-807.

45. Kaneda T, Honda A, Hakozaki A, Fuse T, Muto A, Yoshida T. An improved graves' disease model established by using in vivo electroporation exhibited long-term immunity to hyperthyroidism in BALB/c mice. Endocrinology. 2007;148:2335-44.

46. Wu H-J, Ivanov I, Darce J, Hattori K, Shima T, et al. Gut-residing segmented filamentous bacteria drive autoimmune arthritis via Thelper 17 cells. Immunity. 2010;32:815-27.

\section{Ready to submit your research? Choose BMC and benefit from:}

- fast, convenient online submission

- thorough peer review by experienced researchers in your field

- rapid publication on acceptance

- support for research data, including large and complex data types

- gold Open Access which fosters wider collaboration and increased citations

- maximum visibility for your research: over $100 \mathrm{M}$ website views per year

At BMC, research is always in progress.

Learn more biomedcentral.com/submissions 